Relations industrielles

Industrial Relations

\title{
The Big Business Executive. By Mabel Newcomer. New York: Columbia University, 1955. 164 pp. \$4.25.
}

\section{Rodrigue Giroux}

Volume 13, numéro 2, avril 1958

URI : https://id.erudit.org/iderudit/1022454ar

DOI : https://doi.org/10.7202/1022454ar

Aller au sommaire du numéro

Éditeur(s)

Département des relations industrielles de l’Université Laval

ISSN

0034-379X (imprimé)

1703-8138 (numérique)

Découvrir la revue

Citer ce compte rendu

Giroux, R. (1958). Compte rendu de [The Big Business Executive. By Mabel Newcomer. New York: Columbia University, 1955. 164 pp. \$4.25.] Relations industrielles / Industrial Relations, 13(2), 239-240.

https://doi.org/10.7202/1022454ar

Tous droits réservés (C Département des relations industrielles de l’Université Laval, 1958
Ce document est protégé par la loi sur le droit d'auteur. L'utilisation des services d'Érudit (y compris la reproduction) est assujettie à sa politique d'utilisation que vous pouvez consulter en ligne.

https://apropos.erudit.org/fr/usagers/politique-dutilisation/ 
remarquer Carl. R. Rogers et F. J. Roethlisberger, le patron, le meneur, ne conçoit pas qu'il arrête le flot des communications par sa tendance compulsive à évaluer, à approuver ou désapprouver les autres sans les écouter ni les comprendre.

Marrow consacre plusieurs chapitres à expliquer cette attitude négative du «leader». Il l'illustre avec plusieurs exemples concrets. Mais comment changer l'attitude du 《leader»; comment surtout l'amener à comprendre ses pro. pres faiblesses? Marrow propose la méthode des conférences. Chaque conférence est analysée avec les techniques suivantes:

a) la formule de réaction: «PostMeeting Reaction Form »;

b) l'indice de participation;

c) l'observateur indépendant;

d) l'enregistrement sur bande sonore;

e) la discussion circulaire.

Marrow parle beaucoup de participation. Il montre comment de nombreux problèmes furent solutionnés à Harwood en obtenant la participation des employés. Il en loue les effets constamment, mais il ignore un fait important. Si la participation a pour but véritable de manipuler les employés afin de leur faire accepter des transformations auxquelles ils s'opposent par principe, à ce moment l'échec est inévitable. Les malaises qui tourmentent l'organisation ne peuvent pas être éliminés s'ils résultent de l'incompétence de la gérance.

La thèse de Marrow est vivante; elle essaie de solutionner un problème aigu. Toutefois, elle y aurait beaucoup gargné si elle avait été exposée de façon plus systématique et plus vigoureuse. Il aurait été préférable d'énoncer d'abord les principes psychologiques et de les expliquer ensuite par des exemples. L'auteur a probablement voulu vulgariser la science psychologique afin d'en rendre les principes plus acceptables pour les patrons et les meneurs de l'industrie. L'effet a peut-être été manqué, car l'exposé de sa doctrine est trop souvent interrompu par des études de cas. Ceux-ci, quoique bien choisis, sont évidemment trop nombreux. Il en est de même de tout bon vin: quand on en boit trop, il perd de sa saveur.

Rodrigue Giroux
The Big Business Executive. By Mabel Newcomer. New York: Columbia University, 1955. 164 pp. \$4.25.

Cet entrefilet a paru dans la page financière d'un journal quotidien bien connu: «M. Joseph Dupont a été élu à la présidence de la Compagnie Durand \& Durand, Ltée. Il assumera également les fonctions de président du conseil d'administration ». Plusieurs lecteurs se demanderont comment $\mathbf{M}$. Dupont est parvenu à obtenir une telle position. Le livre de Newcomer fut spécialement conçu pour répondre à cette question.

Jusqu'à date, la plupart des études sur la position de président et de viceprésident ont surtout porté sur la nature de la position, c'est-à-dire sur les fonctions impliquées. Seulement quelquesunes ont scruté les origines sociales de nos meneurs d'entreprises depuis 1900 . Quelle était leur expérienoe globale, leur entraînement? Quel niveau d'éducation ont-ils atteint? Leur entrainement et leur éducation répondaient-elles aux exigenoes de la position? Les qualifications requises variaient-elles suivant le genre d'entreprise? Quels sentiers ces nouveaux présidents ont-ils suivis pour atteindre leur but? $\mathrm{Y}$ sont-ils parvenus parce qu'ils étaient les candidats les mieux qualifiés, ou par suite de leur ancienneté et de leur influence familiale? Pour répondre à ces questions, l'auteur a choisi une coupe longitudinale des présidents des grandes entreprises américaines depuis 1898 jusqu'à 1953. Cette coupe, il l'analyse au moyen de trois échantillons couvrant les années $1900,1925,1950$. Ces échantillons in-. cluent les présidents et les vice-présidents des conseils d'administration des. compagnies dont l'actif était de plus de $\$ 75,000,000$ en $1950, \$ 50,000,000$ en 1925 et $\$ 25,000,000$ en 1900 .

L'analyse qui en résulte nous livre des faits intéressants que l'auteur n'ose pas trop commenter. En effet, il se défend bien de vouloir le faire: Le document est court et facile à lire. Il laisse entrevoir à quelle politique sociale l'ont peut s'attendre de l'exécutif de la plupart des compagnies quand leurs présidents répondent à un stéréotype bien défini. Le portrait suivant, quoique basé sur des données moyennes, semble bien définir le président typique bien connu. 
M. J. Dupont a été choisi comme candidat à la présidence par son prédécesseur, qui l'a recommandé personnellement au conseil d'administration. Issu d'une famille blanche anglo-saxonne, M. J. Dupont est surtout épiscopalien ou presbytérien. Ses ancêtres, comme lui-même d'ailleurs, ont toujours été de bons républicains. Son père était connu dans le monde des affaires et il était fortuné, sinon à l'aise. M. Dupont père fut président de la même compagnie, avant son fils. Le nouveau président, M. Joseph Dupont, compléta ses études au collège et obtint un B.A. ou un B.S., surtout en génie ou en droit. (Il est à noter qu'un très grand nombre d'administrateurs sont des salariés professionnels, sortis des rangs de lientreprise dont ils sont maintenant le président). La famille du nouveau président jouissait d'un certain prestige social qui l'aida beaucoup dans son éduoation, mais non dans lobtention de sa première position permanente, comme avocat, administrateur junior ou employé de bureau. Après environ 20 années de service dans la même compagnie dont sept ans comme vice-président, M. Dupont fut élu à la présidence à l'âge moyen de 50 ans. Il avait surtout servi dans le département de l'opération, c'est-à-dire de la production, et dans le département légal. Normalement, il devrait demeurer à son nouveau poste pour 15 à 20 ans. Ayant atteint l'âge de 70 ans, il prendra sa retraite, jouissant d'un fonds de pension spécial et possédant des actions privilégiées. Quels sont les facteurs qui ont amené M. Dupont à accepter la présidence de la Compagnie? Ce sont: le salaire, environ $\$ 100,000$ par année, la sécurité de l'emploi et le prestige de la position.

Ce profil tiré du volume de Mabel Newoomer nous déçoit quelque peu. Une question importante sungit immédiatement à l'esprit. Si l'on considère tous les facteurs qui ont aidé directement ou indirectement les présidents dans l'obtention de leur poste, quels sont les faits qui nous prouvent la qualité du choix fait par les conseils d'administration? Oublions le document de l'auteur afin de conjecturer sur l'éducation et la formation des futurs présidents de nos grandes entreprises industrielles et commerciales. En 1953, seulement $8 \%$ des employeurs désiraient embaucher des gradués en arts libéraux. La majorité recherchait des ingénieurs. On peut en déduire que d'ici vingt ans la majorité des présidents seront des ingénieurs. En présence des problèmes sociaux qui troublent notre monde capitaliste, ces hommes à formation technique pourront-ils saisir l'ampleur des problèmes, pourront-ils poser les actes qui s'imposent? La recherche de Newoomer ne va pas jusque-là, mais du moins elle nous livre matière à discussion.

\section{Rodrigue Giroux}

Human Relations for Management. By Edward C. Bursk, Edit. New York: Harper \& Bros., 1956, 372 pp. \$5.00.

Cette septième édition de Human Relations for Management présente une foule d'idées qui ne sont peut-être pas nouvelles mais qui prennent de plus en plus d'emprise sur la philosophie de ladministration du personnel. Quand un éditeur compte parmi ses collabonateurs des noms tels que Wendell Johnson, F.J. Roethlisberger, Chris Argyris, Robert L. Katz, Charles R. Walker et Solomon Barkin, le lecteur peut s'attendre à des exposés, sinon récents, du moins intéressants. En effet, plusieurs chapitres du volume sont des articles ou des extraits de travaux qui ont déjà été publiés. On y parle de tout, depuis une philosophie du capitalisme jusquau génie industriel.

Après une lecture attentive, plusieurs se demanderont, mais qu'est-ce qu? c'est les relations humaines? Depuis des années, les chercheurs ont essayé par tous les moyens de rétablir les communications entre le patron et l'ouvrier. D'aucuns ont mis au point des techniques en relations humaines qui, en cristállisant les problèmes étudiés, les ont complètement faussés, car ces techniques ne parvenaient pas à saisir toute la dynamique humaine. D'autres. voyant le problème, se sont lancés dans des énoncés philosophiques sur les relations humaines, oubliant complètement que l'homme vit dans le concret, dans un milieu qui le presse de toutes narts avec des demandes relativement importantes mais souvent en conflit. Trop de chercheurs ont oublié de bien situer l'importance relative des relations humaines dans le comportement du 